\title{
Limites térmicos para a germinação em função da origem de sementes de espécies de Eugenia (Myrtaceae) nativas do Brasil
}

Edmir Vicente Lamarca ${ }^{1,4}$, Cristiana Vendrame e Silva ${ }^{2}$ e Claudio José Barbedo ${ }^{3}$

Recebido em 25/10/2010. Aceito em 16/03/2011

\begin{abstract}
RESUMO
(Limites térmicos para a germinação em função da origem de sementes de espécies de Eugenia (Myrtaceae) nativas do Brasil). A germinação das sementes é dependente de numerosos fatores abióticos, dentre os quais a temperatura figura entre os principais. No presente estudo analisou-se o comportamento germinativo de sementes de E. brasiliensis, E. involucrata, E. pyriformis e E. uniflora em resposta a diferentes condições de luz e temperatura, incluindo-se o cálculo da soma térmica durante o desenvolvimento e maturação das sementes. Os resultados demonstraram que a germinação de sementes e o desenvolvimento de plântulas normais ocorreram adequadamente na faixa de $20{ }^{\circ} \mathrm{C}$ a $30^{\circ} \mathrm{C}$. Nessa faixa, as sementes foram indiferentes à luz e à alternância de temperatura para a germinação. Especificamente para Eugenia pyriformis, avaliaram-se temperaturas superiores a $30^{\circ} \mathrm{C}$ e inferiores a $20^{\circ} \mathrm{C}$, verificando-se que as sementes germinaram na faixa térmica de $10{ }^{\circ} \mathrm{C}$ a $35^{\circ} \mathrm{C}$, mas não a $5^{\circ} \mathrm{C}$ e a $40{ }^{\circ} \mathrm{C}$; os maiores valores de germinação e de IVG foram observados a $25^{\circ} \mathrm{C}$ e $30^{\circ} \mathrm{C}$. Ficou evidente que a secagem modifica os limites e exigências térmicas para a germinação das sementes de E. pyriformis, uma vez que os maiores níveis de desidratação resultaram em maiores prejuízos à germinação fora da faixa térmica ótima. As sementes de E. pyriformis coletadas em Lavras, $\mathrm{MG}$, que receberam maior quantidade de graus-dia durante o desenvolvimento e a maturação, apresentaram melhor desempenho em relação às sementes coletadas em Campinas, SP e em São Paulo, SP.
\end{abstract}

Palavras-chave: graus-dia, luz, Myrtaceae, temperatura

\begin{abstract}
(Thermal requirements for the seeds of Brazilian species of Eugenia (Myrtaceae) according to their origin). Seed germination is dependent on several abiotic factors including temperature. In this study, we analyzed the germination of seeds of Eugenia brasiliensis, E. involucrata, E. pyriformis and E. uniflora in response to different conditions of light and temperature. Seeds of all species showed high germination values from 20 to $30^{\circ} \mathrm{C}$. Germination of seeds of E. Pyriformis was also evaluated at temperatures higher than $30^{\circ} \mathrm{C}$ and lower than $20^{\circ} \mathrm{C}$. The germination and the normal seedling development occurred from $10^{\circ} \mathrm{C}$ to $35^{\circ} \mathrm{C}$, not at $5^{\circ} \mathrm{C}$ and $40^{\circ} \mathrm{C}$. Results also showed that desiccation changed the limits for germination in E. Pyriformis since the most dehydrated seeds had the highest damaged in germination at suboptimal temperatures. Thermal time during development and maturation of seeds was also considered. The seeds of E. pyriformis from Lavras, MG, which received the greatest number of degree-days during development and maturation, showed better performance than the seeds from Campinas, SP and São Paulo, SP.
\end{abstract}

Key words: degree-day, light, Myrtaceae, temperature

\section{Introdução}

A regeneração da maioria das espécies arbóreas em florestas tropicais ocorre por intermédio de sementes. Dessa forma, o conhecimento de aspectos ecológicos e fatores ambientais sobre os mecanismos envolvidos na germinação pode contribuir para a compreensão da distribuição geográfica das espécies (Harper 1977; Vázquez-Yanes \& Orozco-Segovia 1990).
A germinação das sementes é dependente de numerosos fatores abióticos, como luz, temperatura, disponibilidade hídrica e concentração de oxigênio (Mayer \& PoljakoffMayber 1989). A luz nem sempre é um fator limitante para a germinação e, para algumas espécies, essa germinação pode ainda ser fortemente influenciada pela temperatura (Smith 1975). O estudo da dependência da temperatura na germinação de uma espécie inclui a determinação da amplitude térmica, da temperatura ótima e das temperatu-

\footnotetext{
Instituto de Botânica, Programa de Pós-Graduação em Biodiversidade Vegetal e Meio Ambiente, São Paulo, SP, Brasil

Incotec América do Sul Ltda. Tecnologia em Sementes, São Paulo, SP, Brasil

Instituto de Botânica, Núcleo de Pesquisa em Sementes. Bolsista CNPq, São Paulo, SP, Brasil

4 Autor para correspondência: lamarcabio@ig.com.br
} 
ras extremas para a germinação e para o desenvolvimento inicial das plântulas.

Sementes de florestas tropicais do Brasil podem germinar em ampla faixa térmica, dependendo do bioma e da região e parecem apresentar uma relação positiva entre a temperatura ótima e o regime térmico da região de sua ocorrência natural (Borghetti 2005). A faixa térmica adequada para a germinação de sementes de espécies tropicais está, frequentemente, entre 20 e $30^{\circ} \mathrm{C}$ (Borges \& Rena 1993; Bilia et al. 1998; Santos et al. 2004; Mello \& Barbedo 2007; Stockman et al. 2007; Coelho et al. 2008; Cardoso \& Pereira 2009; Pires et al. 2009; Pimenta et al. 2010). Contudo, podem ocorrer variações até dentro da mesma espécie, provavelmente devido à variação de fatores ambientais, principalmente a temperatura do ar, tipicamente relatada como acúmulo de graus-dia.

A família Myrtaceae, com grande ocorrência nas regiões tropicais e subtropicais do mundo, é representada por mais de 1000 espécies. O gênero Eugenia, com mais de uma centena de espécies, compreende algumas nativas do Brasil de grande importância econômica, como Eugenia brasiliensis Lam. (grumixama), Eugenia involucrata DC., (cereja-dorio-grande), Eugenia pyriformis Camb. (uvaia) e Eugenia uniflora L. (pitanga). São espécies que produzem frutos carnosos, de coloração amarela e vermelha, geralmente com poucas sementes (1-3). A dispersão de suas sementes, na maioria das vezes, ocorre por aves e mamíferos (Gressler et al. 2006) e as sementes apresentam comportamento recalcitrante, com diferentes níveis de tolerância à dessecação (Delgado \& Barbedo 2007).

Apesar de grande representatividade e importância ecológica do gênero Eugenia, há poucas informações sobre a germinação de suas sementes. No presente estudo analisouse o comportamento germinativo de sementes de E. brasiliensis, E. involucrata, E. pyriformis e E. uniflora em resposta a diferentes condições de luz e temperatura, incluindo-se $\mathrm{o}$ cálculo da soma térmica durante o desenvolvimento e maturação das sementes.

\section{Material e métodos}

\section{Material vegetal}

Sementes de Eugenia brasiliensis Lam., Eugenia involucrata DC., Eugenia pyriformis Camb. e Eugenia uniflora L. foram obtidas de frutos maduros coletados de matrizes localizadas no Instituto de Botânica, São Paulo, SP (233'', $\left.46^{\circ} 37^{\prime} \mathrm{O}\right)$, na Reserva Biológica e Estação Experimental de Mogi-Guaçu, em Mogi-Guaçu, SP (22 $15^{\circ}$ S, $\left.47^{\circ} 10^{\prime} \mathrm{O}\right)$, na Fazenda Santa Elisa, Instituto Agronômico de Campinas, Campinas, SP ( $\left.22^{\circ} 52^{\prime} \mathrm{S}, 47^{\circ} 04^{\prime} \mathrm{O}\right)$ e no município de Lavras, MG $\left(21^{\circ} 13^{\prime} \mathrm{S}, 44^{\circ} 59^{\prime} \mathrm{O}\right)$. As sementes foram extraídas manualmente e armazenadas em câmara fria a $7^{\circ} \mathrm{C}$ até o inicio dos experimentos, não excedendo sete dias (Barbedo et al. 1998; Andrade \& Ferreira 2000).

\section{Determinações físicas}

As sementes foram caracterizadas, inicialmente, quanto ao teor de água e conteúdo de massa seca. Ambos foram determinados gravimetricamente, pelo método de estufa a $103{ }^{\circ} \mathrm{C}$ por 17 horas (ISTA 1985), sendo os resultados apresentados em porcentagem, em base úmida, para teor de água e em g.semente ${ }^{-1}$, para massa seca (Brasil 2009).

\section{Temperatura e luz na germinação}

Para este experimento, foram utilizadas sementes de E. brasiliensis, E. involucrata, E. pyriformis e E. uniflora colhidas no Instituto de Botânica. O teste de germinação foi conduzido em caixas do tipo gerbox, transparentes para os tratamentos com presença de luz e pretos para os de ausência de luz. O preenchimento dessas caixas foi com vermiculita, umedecida com $75 \mathrm{ml}$ de água. As sementes foram submetidas a diferentes regimes térmicos e luminosos durante a germinação. Para tanto, foram utilizadas câmaras do tipo BOD reguladas a $20^{\circ} \mathrm{C}, 25^{\circ} \mathrm{C}$ e $30^{\circ} \mathrm{C}$, para temperaturas constantes e $20-30^{\circ} \mathrm{C}$ e $20-35^{\circ} \mathrm{C}$, para alternadas.

As avaliações de germinação foram realizadas semanalmente, durante 80 dias sendo registradas as sementes que emitiram raiz primária (para o cálculo de germinação) e as que produziram plântulas normais (para o cálculo de desenvolvimento de plântulas normais). Foi calculado, ainda, o índice de velocidade de germinação (IVG), conforme Maguire (1962). No caso dos tratamentos de escuro, as avaliações foram realizadas sob luz verde de segurança (Amaral-Baroli \& Takaki 2001).

\section{Alterações na exigência térmica para germinação em função do material vegetal}

Sementes de E. pyriformis, coletadas em Mogi-Guaçu, foram separadas em função do estádio de dispersão, sendo então colocadas para germinar em diferentes temperaturas. Sementes coletadas diretamente das árvores, de frutos maduros foram classificadas como pré-dispersas, enquanto as coletadas após queda natural, como recém dispersas. $\mathrm{O}$ teste de germinação foi realizado nos cinco regimes térmicos descritos anteriormente.

Em uma segunda colheita (Instituto de Botânica, São Paulo, SP), sementes recém dispersas foram submetidas a dois níveis de secagem, utilizando-se estufa com circulação forçada de ar; as sementes foram dispostas em prateleiras forradas com tela de polietileno, em camadas simples sem sobreposição (Delgado \& Barbedo 2007). A secagem foi intermitente, com 10 horas a $40 \pm 1{ }^{\circ} \mathrm{C}$ seguidas de 14 horas de repouso. Neste último ciclo, a temperatura mínima atingida foi $25^{\circ} \mathrm{C}$. Este processo foi repetido até que as sementes atingissem $60 \%$ e $53 \%$ de água. O teste de germinação das sementes desses dois teores de água foi realizado em câmaras do tipo BOD reguladas para 5, 10, 15, 20, 25, 30, 35 e 40 ${ }^{\circ} \mathrm{C}$, na ausência de luz, com as sementes colocadas em rolo 
de papel germitest, com duas folhas para base e uma para cobertura (Brasil 2009).

\section{Soma térmica na germinação}

Sementes de E. pyriformis de Lavras, MG, Campinas, SP e São Paulo, SP foram colocadas para germinar sob oito regimes térmicos $\left(5,10,15,20,25,30,35 \mathrm{e} 40^{\circ} \mathrm{C}\right)$. O teste de germinação foi conduzido conforme descrito anteriormente para o estudo de secagem na germinação.

A soma térmica foi calculada em graus-dia, acumulados desde a antese até a dispersão natural das sementes. Para tanto, inicialmente inflorescências foram marcadas no período de máximo florescimento da espécie. Os frutos e sementes foram colhidos imediatamente após a dispersão, quando apresentaram coloração característica para a espécie.

O somatório de graus-dia (GD) foi realizado desde a antese até a coleta, segundo as seguintes equações, propostas por Villa Nova et al. (1972):

$$
\begin{aligned}
& \mathrm{GD}=\left(\mathrm{T}_{\mathrm{m}}-\mathrm{T}_{\mathrm{b}}\right)+\left(\mathrm{T}_{\mathrm{M}}-\mathrm{T}_{\mathrm{m}}\right) / 2, \text { para } \mathrm{T}_{\mathrm{m}}>\mathrm{T}_{\mathrm{b}} ; \\
& \mathrm{GD}=\left(\mathrm{T}_{\mathrm{M}}-\mathrm{T}_{\mathrm{b}}\right)^{2} / 2\left(\mathrm{~T}_{\mathrm{M}}-\mathrm{T}_{\mathrm{m}}\right), \text { para } \mathrm{T}_{\mathrm{m}}<\mathrm{T}_{\mathrm{b}} \mathrm{e} \\
& \mathrm{GD}=0 \text {, para } \mathrm{T}_{\mathrm{b}}>\mathrm{T}_{\mathrm{M}} \cdot \\
& \text { Em que: } \\
& \mathrm{GD}=\text { graus-dia }\left({ }^{\circ} \mathrm{C} \mathrm{d}\right) ; \\
& \mathrm{T}_{\mathrm{M}}=\text { temperatura máxima diária }\left({ }^{\circ} \mathrm{C}\right) ; \\
& \mathrm{T}_{\mathrm{m}}=\text { temperatura mínima diária }\left({ }^{\circ} \mathrm{C}\right) \mathrm{e} \\
& \mathrm{T}_{\mathrm{b}}=\text { temperatura base }\left({ }^{\circ} \mathrm{C}\right) .
\end{aligned}
$$

Os graus-dia acumulados foram calculados considerando-se a temperatura base de $10{ }^{\circ} \mathrm{C}$, conforme descrito em Pedro Júnior (1977). Os dados climáticos foram obtidos das estações meteorológicas da Universidade Federal de Lavras, Lavras, MG, do Instituto Agronômico de Campinas, Campinas, SP e do Instituto Astronômico e Geofísico da Universidade de São Paulo, São Paulo, SP.

\section{Delineamento experimental e procedimento estatístico}

O delineamento experimental para todos os experimentos foi o inteiramente casualizado, em esquema fatorial associando-se luz $\mathrm{x}$ temperatura $(2 \times 5)$, tipo de colheita $\mathrm{x}$ temperatura $(2 \times 5)$, nível de secagem $x$ temperatura $(2 \times$ 8 ) e local de coleta $x$ temperatura $(3 \times 8)$. Os dados obtidos foram submetidos à análise de variância (teste F), ao nível de $5 \%$ de probabilidade. Quando pertinente, as médias foram comparadas entre si pelo teste de Tukey, também ao nível de 5\% (Santana \& Ranal 2004).

\section{Resultados e discussão}

\section{Temperatura e luz na germinação}

A análise de variância para os dados de germinação (não apresentados), plântulas normais (Fig. 1) e IVG (Fig. 2) não apresentou interação significativa entre os fatores luz e temperatura para as sementes de E. brasiliensis, E. involucrata,
E. pyriformis e E. uniflora. A germinação (na faixa de 20 a $30{ }^{\circ} \mathrm{C}$ e nas alternadas $20 / 30$ e $20 / 35^{\circ} \mathrm{C}$ ) não apresentou diferenças significativas, sendo a média geral, para todas as espécies, temperaturas e regimes de luz, de 98\%.

Os resultados demonstraram que as sementes de Eugenia germinam e desenvolvem plântulas normais adequadamente na faixa de temperatura de $20{ }^{\circ} \mathrm{C}$ a 30 ${ }^{\circ} \mathrm{C}$ e que são indiferentes à luz e à alternância de temperatura. Contudo, a $30{ }^{\circ} \mathrm{C}$ as sementes de E. brasiliensis e de E. uniflora germinaram mais rapidamente que nas demais temperaturas (Fig. 2A, D). Essa faixa térmica (20 ${ }^{\circ} \mathrm{C}$ a $30{ }^{\circ} \mathrm{C}$ ) também foi adequada para a germinação de sementes de outras espécies de Eugenia, como E. rostrifolia Legr, (Santos et al. 2004) e E. pleurantha O. Berg. (Masetto et al. 2009). Limites térmicos similares para a germinação de sementes de diferentes espécies dentro do mesmo gênero também foram observadas em Caesalpinia (Ferraz-Grande \& Takaki 2006; Mello \& Barbedo 2007), Phoenix (Iossi et al. 2003; Pimenta et al. 2010) e Tabebuia (Oliveira et al 2005; Stockman et al 2007), sugerindo uma forte determinação filogenética.

\section{Alterações na exigência térmica para germinação em função do material vegetal}

Não houve interação significativa entre estádio de dispersão e temperaturas de germinação para as variáveis germinação, desenvolvimento de plântulas normais e índice de velocidade de germinação. As sementes de Eugenia pyriformis recém dispersas apresentaram valores ligeiramente mais elevados para desenvolvimento de plântulas normais e para índice de velocidade de germinação (respectivamente, $98 \%$ e 0,554 ) do que as pré-dispersas (respectivamente, $94 \%$ e 0,517$)$.

Considerando-se as médias dos dois estádios de dispersão, verificou-se que a secagem das sementes de $E$. pyriformis, levando o teor de água de $68 \%$ para $60 \%$ e $53 \%$, causou danos na germinação e no desenvolvimento (Fig. 3B, D), semelhante ao verificado em sementes de Eugenia involucrata DC. (Barbedo et al. 1998). De acordo com Delgado \& Barbedo (2007), sementes de Eugenia são sensíveis à secagem; contudo, verificou-se existirem níveis distintos de tolerância à dessecação entre as sementes das diferentes espécies desse gênero, as de E. pyriformis estando entre as mais sensíveis. Ainda segundo esses autores, sementes de $E$. pyriformis iniciam a perda da viabilidade em teor de água superior e próximo a $65 \%$, conforme também verificado no presente estudo.

Em relação às exigências térmicas, verificou-se que houve prejuízo principalmente para a velocidade da germinação (Fig. 3F). Além disso, ficou evidente que esse prejuízo foi maior quando as sementes foram submetidas à maior secagem. Assim, na temperatura de $25^{\circ} \mathrm{C}$ a desidratação das sementes não causou tanto dano quando comparada, por exemplo, com $15{ }^{\circ} \mathrm{C}$ (Fig. 3B). Considerando-se todas 

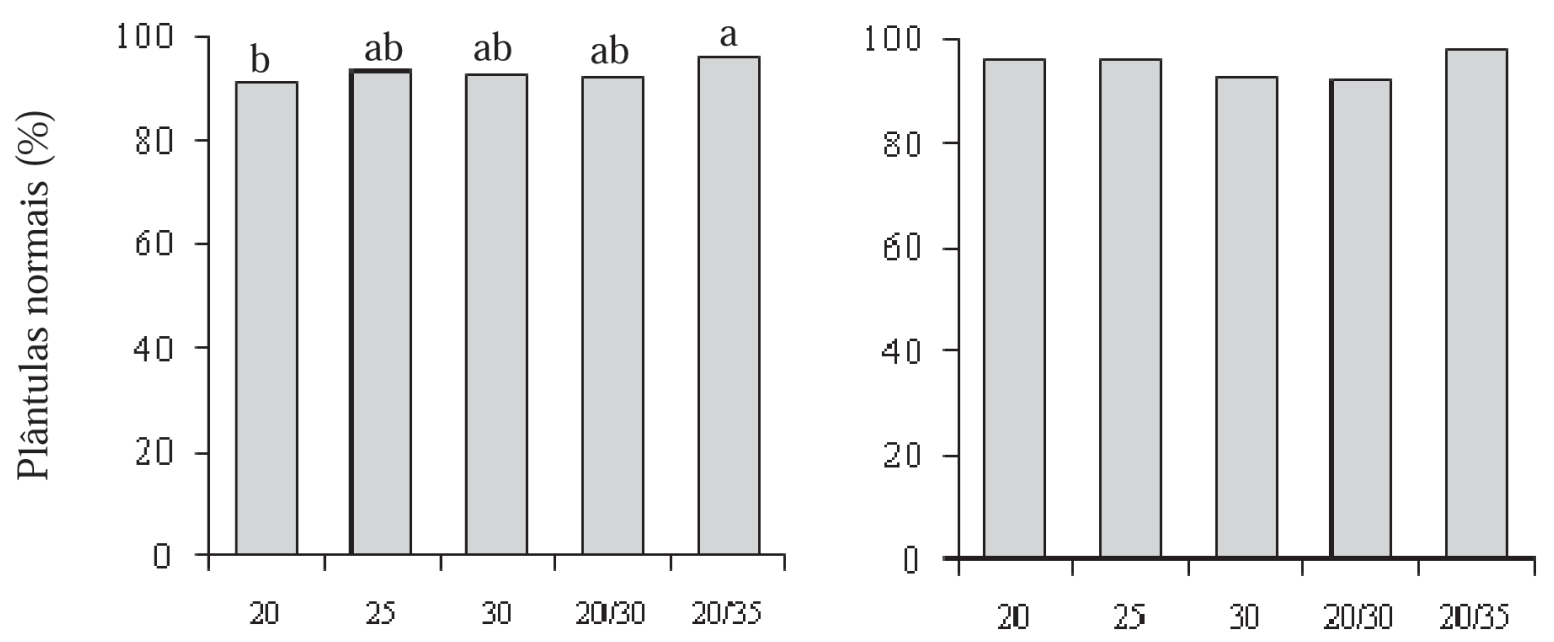

C

D
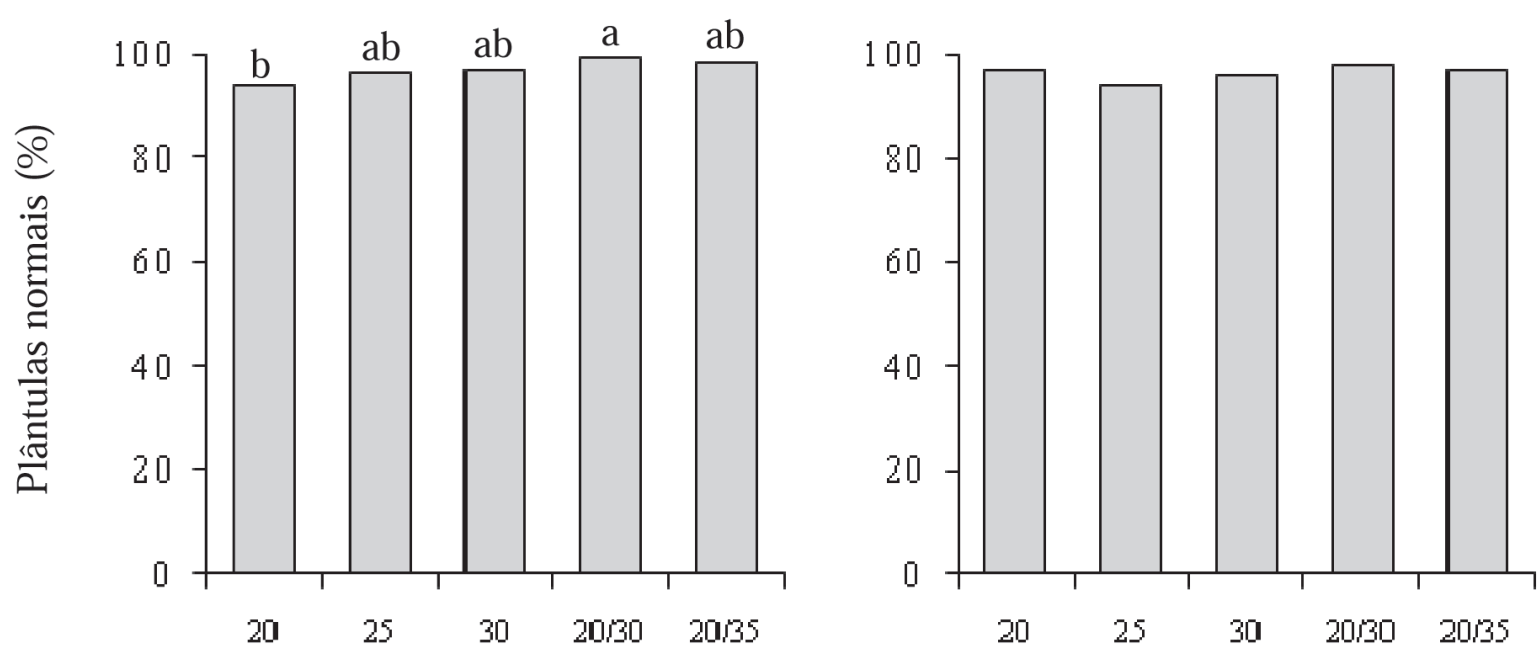

Regimes térmicos $\left({ }^{\circ} \mathrm{C}\right)$

Figura 1. Desenvolvimento de plântulas normais durante o teste de germinação de sementes de E. brasiliensis (A), E. involucrata (B), E. pyriformis (C) e E. uniflora (D), sob diferentes regimes térmicos. Colunas com a mesma letra ou sem letras não apresentam diferenças significativas (Tukey $5 \%$ ).

as faixas térmicas estudadas, as temperaturas limítrofes, respectivamente máxima e mínima, parecem estar entre $5^{\circ} \mathrm{C}$ e $10^{\circ} \mathrm{C}$ e entre $35^{\circ} \mathrm{C}$ e $40^{\circ} \mathrm{C}$ (Fig. $3 \mathrm{~B}, \mathrm{D}, \mathrm{F}$ ), uma vez que a 5 e a $40{ }^{\circ} \mathrm{C}$ não houve resposta germinativa. Nas duas situações de dispersão e nos dois níveis de secagem a faixa térmica considerada adequada para germinação foi a mesma, ou seja, entre $25^{\circ} \mathrm{C}$ e $30^{\circ} \mathrm{C}$.

\section{Soma térmica na germinação}

A análise de variância apresentou interação significativa entre locais de coleta e temperaturas, para os dados de germinação, plântulas normais e IVG (Fig. 4).
$\mathrm{Na}$ faixa de $15^{\circ} \mathrm{C}$ a $30^{\circ} \mathrm{C}$ não houve diferenças significativas, para germinação, entre as regiões de coleta das sementes (Fig. 4A), mas houve para desenvolvimento de plântulas normais e para IVG (Fig. 4B, C). Entre as regiões observou-se melhor desempenho para as sementes de Lavras, principalmente para plântulas normais e IVG. Tal comportamento pode ter ocorrido em função dos graus-dia acumulados durante o desenvolvimento e maturação. Pela Tab. 1 nota-se que as sementes de Lavras receberam 495 graus-dia (GD) durante o desenvolvimento e maturação, cerca de $40 \mathrm{GD}$ a mais que as sementes de Campinas e São Paulo, que receberam respectivamente 456 e 458 GD (Tab. 1). O aumento no somatório de GD provavelmente 

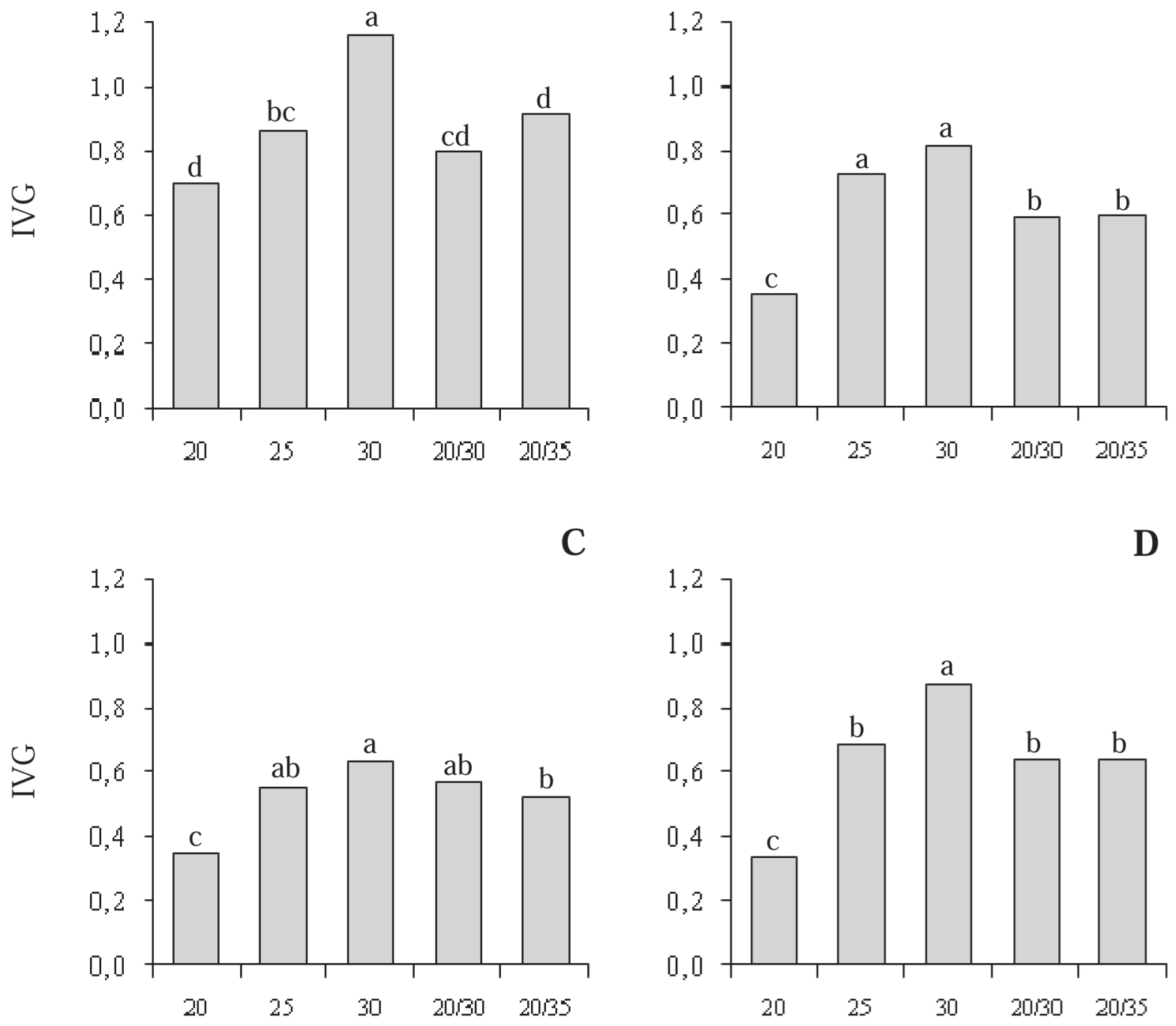

\section{Regimes térmicos $\left({ }^{\circ} \mathrm{C}\right)$}

Figura 2. Índice de velocidade de germinação (IVG) de sementes de E. brasiliensis (A), E. involucrata (B), E. pyriformis (C) e E. uniflora (D), sob diferentes regimes térmicos. Colunas com a mesma letra não apresentam diferenças significativas (Tukey 5\%).

proporcionou maiores valores no acúmulo de matéria seca e leve redução no teor de água para as sementes de Lavras em relação às demais (Tab. 1). Essas relações também foram observadas por Daws et al. (2004; 2006), correspondendo com às fases II e III do processo de desenvolvimento de sementes, caracterizadas pelo acúmulo de reservas e aumento progressivo da massa seca, seguido de redução no teor de água (Castro et al. 2004).

Os maiores valores de germinação apresentados pelas sementes de Lavras nas temperaturas extremas podem estar associados à adaptabilidade da espécie às flutuações térmicas da região e aos maiores valores de massa seca. Estudos com sementes de Aesculus hippocastanum L. e
Acer pseudoplatanus L. coletadas em diferentes localidades demonstraram que há estreita relação entre as flutuações térmicas e a temperatura basal e, também, entre o somatório de GD e o aumento de massa seca (Daws et al. 2004; 2006). Há, ainda, relação entre a massa seca e a velocidade de germinação (Norden et al. 2009). Os resultados apresentados por Daws et al. $(2004 ; 2006)$, associados com os resultados apresentados pelas sementes de Eugenia pyriformis neste trabalho, demonstram que as condições ambientais, principalmente a temperatura do ar, exercem grande influência nas características germinativas das sementes.

As sementes de E. brasiliensis, E. involucrata, E. pyriformis e E. uniflora coletadas na mesma região apresentaram 
A
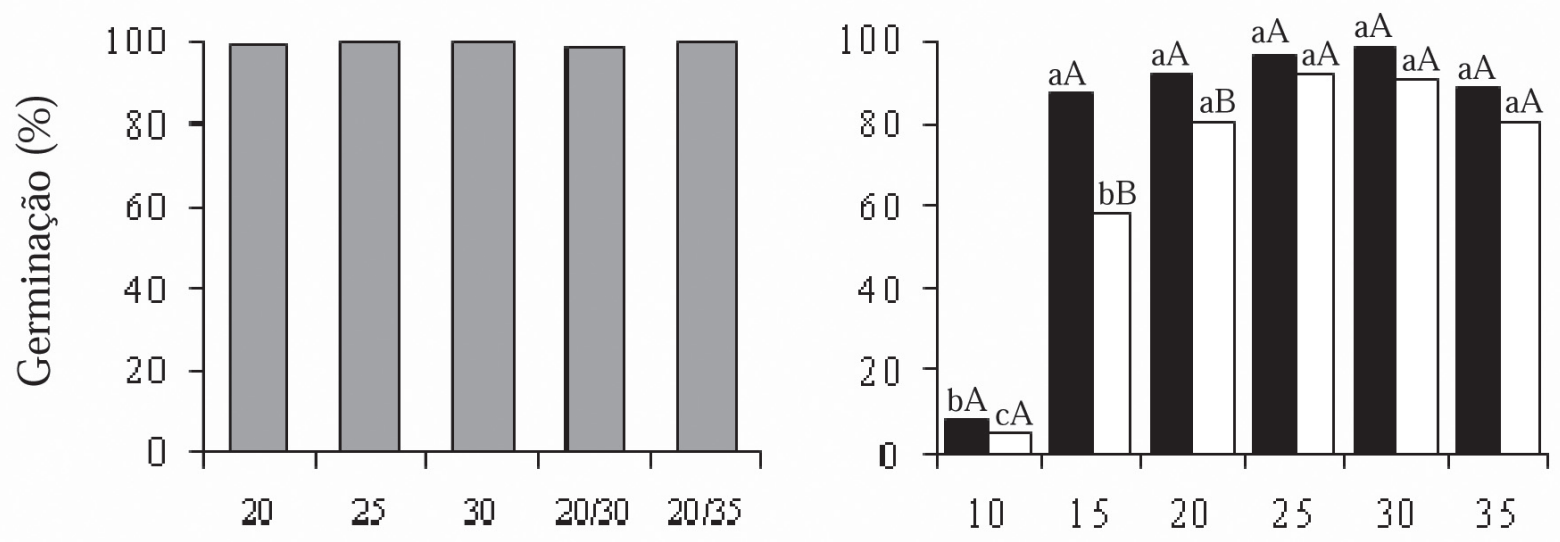

C

D
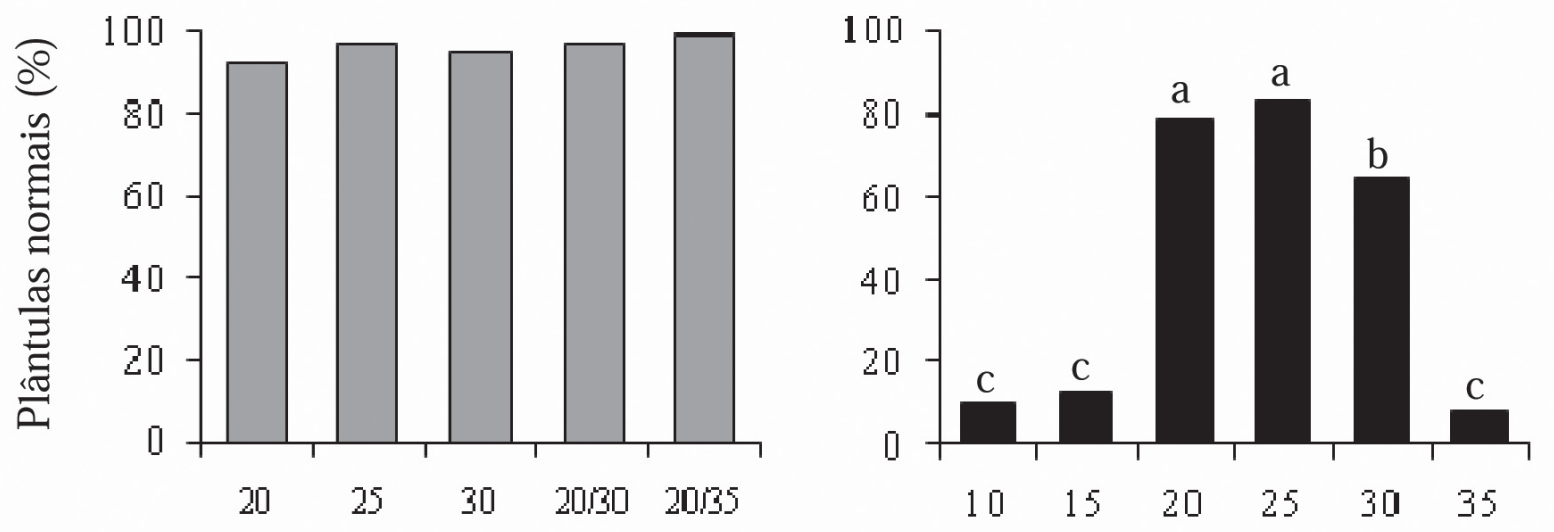

$\mathbf{E}$
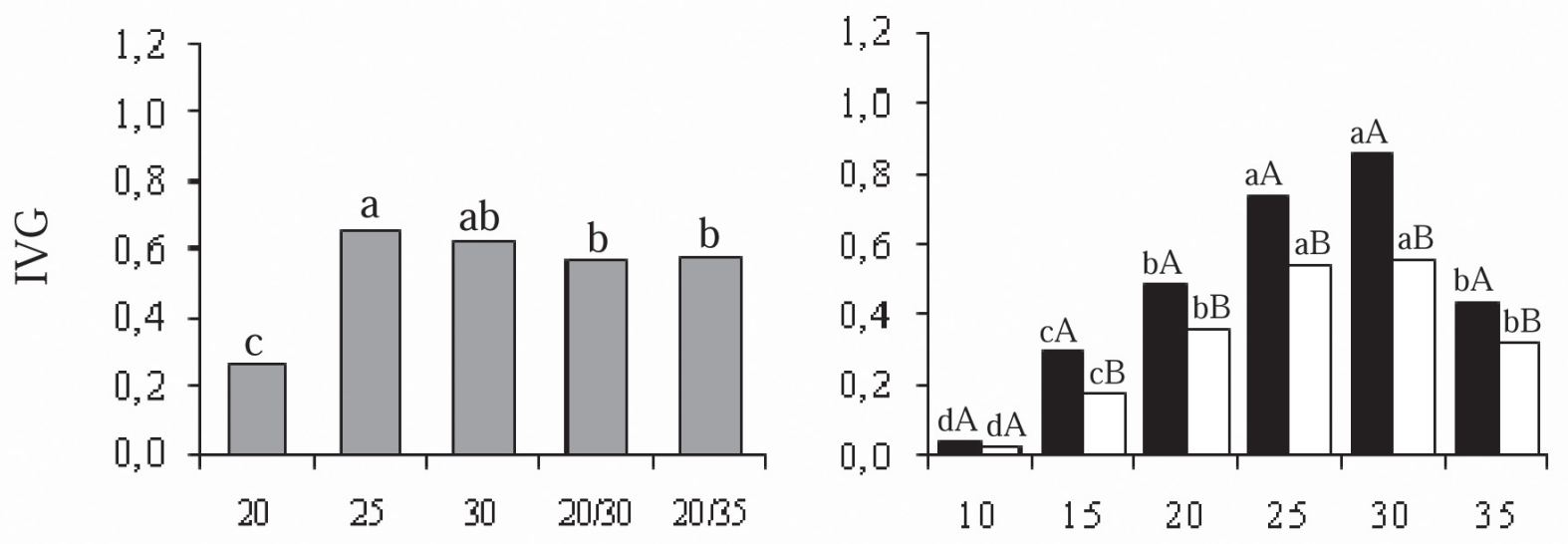

Regimes térmicos $\left({ }^{\circ} \mathrm{C}\right)$

Figura 3. Germinação de sementes (A e B), desenvolvimento de plântulas normais (C e D) e índice de velocidade de germinação (E e F) de Eugenia pyriformis em função do regime térmico e do grau de secagem. A, C e E: médias obtidas para as diferentes temperaturas, considerando-se os dois estádios de dispersão das sementes, com $68 \%$ de água (colunas em cinza); B, D e F: sementes submetidas a dois níveis de secagem (colunas pretas - até $60 \%$ de água, colunas brancas - até $53 \%$ de água). Médias seguidas pela mesma letra (minúscula compara temperaturas e maiúscula compara grau de secagem) ou sem letras não diferem entre si (Tukey 5\%). 

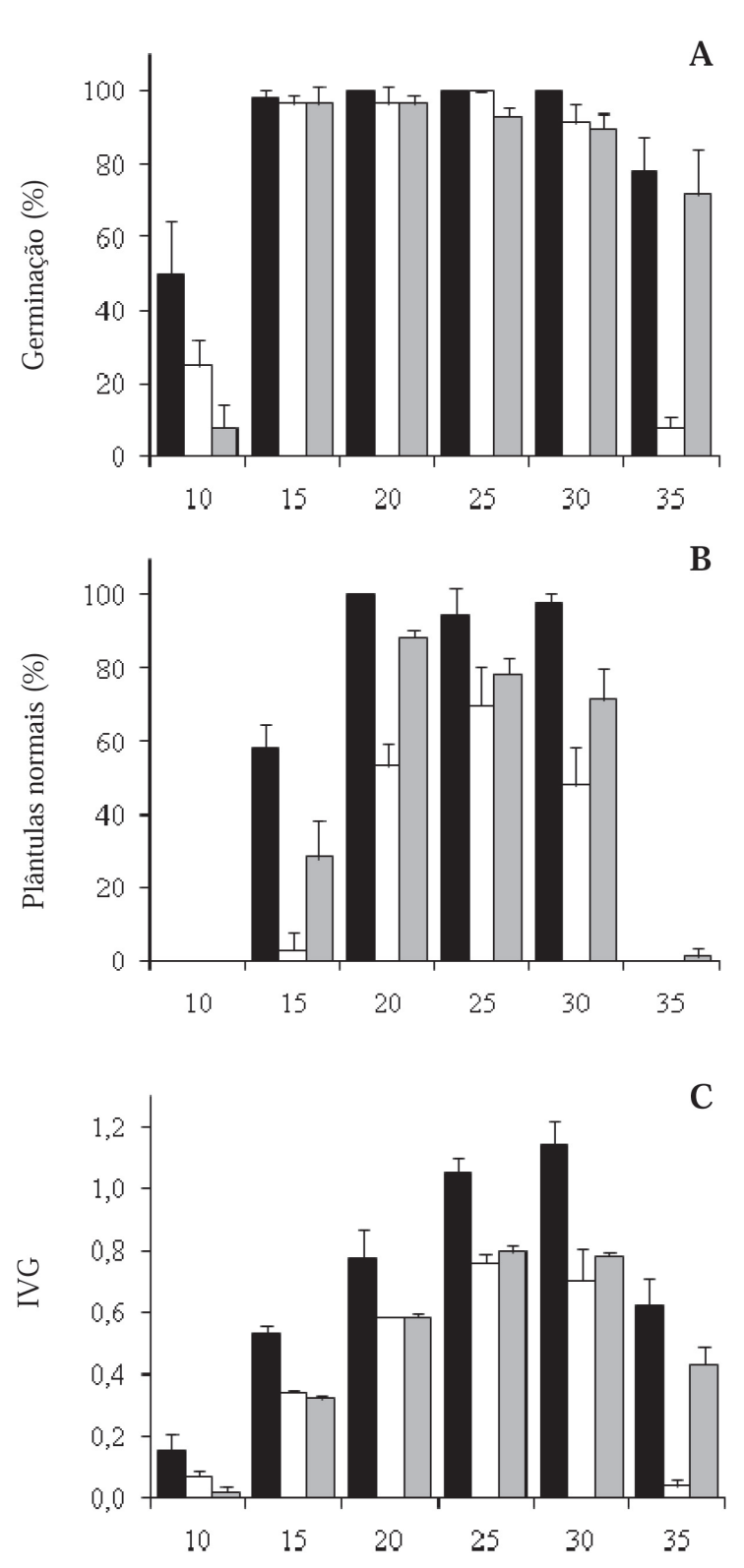

Regimes térmicos $\left({ }^{\circ} \mathrm{C}\right)$

Figura 4. Germinação de sementes (A), desenvolvimento de plântulas normais (B) e índice de velocidade de germinação (C) de Eugenia pyriformis coletadas em Lavras (colunas pretas), Campinas (colunas brancas) e São Paulo (colunas cinzas). As barras representam os valores médios acompanhados do desvio padrão. exigências térmicas mais próximas entre si do que as da mesma espécie (E. pyriformis) coletadas em regiões diferentes. O comportamento germinativo distinto entre populações da mesma espécie e, por outro lado, comum entre espécies da mesma região, sugere que a pressão de seleção do ambiente tenha sido bastante expressiva sobre o potencial germinativo, adaptando as exigências da germinação às condições térmicas do local de origem.

Muitas espécies vegetais exibem diferentes respostas fisiológicas às adversidades impostas pelo seu habitat, que consistem num conjunto de características adaptativas decorrentes da constituição genética influenciada pelas condições ambientais (Daws et al. 2005; Gebler et al. 2005; Renaut et al. 2005). O comportamento germinativo apresentado pelas sementes de Eugenia, no presente trabalho, mostrou-se muito próximo entre as espécies de Eugenia da mesma região (Fig. 1, 2). Por outro lado, notou-se que as sementes da mesma espécie (E. pyriformis) oriundas de regiões distintas, ou de épocas distintas, apresentaram diferentes respostas às variações térmicas para a germinação. A similaridade de comportamento entre espécies do mesmo gênero pode indicar forte determinação filogenética. Contudo, a variabilidade de comportamento das sementes de E. pyriformis oriundas de regiões diferentes sugere grande influência do ambiente sobre essa característica.

\section{Agradecimentos}

Os autores agradecem ao Dr. José Márcio Rocha Faria (UFLA), ao Dr. Edvaldo Aparecido Amaral da Silva (UNESP), a Dra. Priscila Fratin Medina (IAC) e ao Ms. João José Dias Parisi (IAC), pela permissão e auxilio na coleta das sementes; à Dra. Lucia Rossi (IBt) pela identificação das espécies; à Universidade Federal de Lavras, Lavras, MG, Instituto Agronômico de Campinas, Campinas, SP e ao Instituto Astronômico e Geofísico da Universidade de São Paulo, São Paulo, SP, pelo fornecimento dos dados climáticos; ao CNPq pelas bolsas concedidas E.V. Lamarca (Doutorado), C.V. Silva (PIBIC) e C.J. Barbedo (produtividade em pesquisa, Proc. 308045/2007-6) e pelo auxílio financeiro aos projetos (Proc. 481484/2007-8 e Proc.477640/2009-5); à FAPESP, pelo auxílio financeiro ao projeto (Proc. 2005/04139-7); à Bióloga Débora Manzano Molizane pelo auxílio na confecção das figuras.

Tabela 1. Localização geográfica, período de maturação, graus-dia acumulados e teor de água e massa seca inicial de sementes de Eugenia pyriformis Camb. coletadas em Lavras, Campinas e São Paulo no ano de 2009. Médias seguidas pela mesma letra não diferem entre si pelo teste de Tukey, a 5\%.

\begin{tabular}{|c|c|c|c|c|}
\hline Localização & Período de maturação & Graus-dia (GD) & Teor de água ( $\%$, base úmida) & $\begin{array}{l}\text { Massa seca } \\
\left(\text { g semente }^{-1}\right)\end{array}$ \\
\hline $\begin{array}{l}\text { Lavras, MG } \\
\left(21^{\circ} 13^{\prime} \mathrm{S}\right)\end{array}$ & 09/08/09 - 23/09/09 & 495 & $60,36 \mathrm{~b}$ & $0,592 \mathrm{a}$ \\
\hline $\begin{array}{l}\text { Campinas, SP } \\
\left(22^{\circ} 52^{\prime} \mathrm{S}\right)\end{array}$ & 20/07/09 - 08/09/09 & 456 & $62,65 \mathrm{ab}$ & $0,315 \mathrm{~b}$ \\
\hline $\begin{array}{l}\text { São Paulo, SP } \\
\left(23^{\circ} 39^{\prime} S\right)\end{array}$ & $25 / 08 / 09-12 / 10 / 09$ & 458 & $66,39 \mathrm{a}$ & $0,207 \mathrm{c}$ \\
\hline Coeficiente de variação (\%) & & & 3,441 & 7,432 \\
\hline
\end{tabular}




\section{Referências bibliográficas}

Amaral-Baroli, A. \& Takaki, M. 2001. Phytochrome controls achene germination in Bidens pilosa L. (Asteraceae) by very low fluence response. Brazilian Archives of Biology and Technology 44: 121-124.

Andrade, R.N.B. \& Ferreira, A.G. 2000. Germinação e armazenamento de sementes de uvaia (Eugenia pyriformis Camb.) - Myrtaceae. Revista Brasileira de Sementes 22: 118-125.

Barbedo, C.J.; Kohama, S.; Maluf, A.M. \& Bilia, D.A.C. 1998. Germinação e armazenamento de diásporos de cerejeira (Eugenia involucrata DC. - Myrtaceae) em função do teor de água. Revista Brasileira de Sementes 20: 184-188.

Bilia, D.A.C.; Barbedo, C.J. \& Maluf, A.M. 1998. Germinação de diásporos de canela (Ocotea corymbosa (Meissn.) Mez - Lauraceae) em função da temperatura, do substrato e da dormência. Revista Brasileira de Sementes 20: 189-194.

Borges, E.E.L. \& Rena, A.B. 1993. Germinação de sementes. In: Aguiar, I.B.; Piña- F.C.M. Rodrigues \& Figliolia, M.B. (Orgs.). Sementes florestais tropicais. Brasília, Abrates.

Borghetti, F. Temperaturas extremas e a germinação das sementes. 2005. In: Nogueira, R.M.C et al. (Orgs.) Estresses ambientais: danos e benefícios em plantas. Recife, Universidade Federal Rural de Pernambuco, Imprensa Universitária.

Brasil. 2009. Ministério da Agricultura, Pecuária e Abastecimento. Brasília, Regras para análise de sementes.

Cardoso, V.J.M. \& Pereira, F.J.M. 2009. Dependência térmica da germinação de sementes de Drymaria cordata (L.) Willd. ex Roem. \& Schult. (Cariophyllaceae). Acta Botanica Brasilica 23: 305-312.

Castro, R.D.; Bradford, K.J. \& Hilhorst, H.W.M. 2004. Desenvolvimento de sementes e conteúdo de água. In: Ferreira, A.G. \& Borghetti, F. (Orgs.). Germinação do básico ao aplicado. Porto Alegre, Artmed.

Coelho, M.F.B.; Sales, D.M.; Dombroski, J.L.D.; Azevedo, R.A.B. \& Albuquerque, M.C.F. 2008. Condições de luz e temperatura na germinação de sementes de algodão do campo [Cochlospermum regium (Schrank) Pilger - Bixaceae]. Revista de Biologia Neotropical 5: 23-31.

Daws, M.I.; Cleland, H.; Chmielarz, P.; Gorin, F.; Leprince, O.; Matthews, S.; Mullins, C.E.; Thanos, C.A.; Vandvik, V. \& Pritchard, H.W. 2006. Variable dessication tolerance in Acer pseudoplatanus seeds in relation to developmental conditions: a case of phenotypic recalcitrance? Functional Plant Biology 33: 59-66.

Daws, M.I.; Garwood, N.C. \& Pritchard, H.W. 2005. Traits of recalcitrant seeds in a semi-deciduous tropical forest in Panamá: some ecological implications. Functional Ecology 19: 874-885.

Daws, M.I.; Lydall, E.; Chmielarz, P.; Leprince, O.; Matthews, S.; Thanos, C.A. \& Pritchard, H.W. 2004. Developmental heat sum influences recalcitrant seed traits in Aesculus hippocastanum across Europe. New Phytologist 162: 157-166.

Delgado, L.F. \& Barbedo, C.J. 2007. Tolerância à dessecação de sementes de espécies de Eugenia. Pesquisa Agropecuária Brasileira 42: 265-272.

Ferraz-Grande, F.G.A. \& Takaki, M. 2006. Efeito da luz, temperatura e estresse de água na germinação de sementes de Caesalpinia peltophoroides Benth. (Caealpinoideae). Bragantia 65: 37-42.

Gebler, A.; Duarte, H.M.; Franco, A.C.; Lüttge, U.; Mattos, E.A.; Nahm, M.; Rodrigues, P.J.F.P.; Scarano, F.R. \& Rennenberg, H. 2005. Ecophysiology of selected tree species in different plant communities at the periphery of the Atlantic forest of SE - Brazil III. Three legume trees in a semi-deciduous dry forest. Trees 19: 523-530.
Gressler, E.; Pizo, M.P. \& Morellato, L.P.C. 2006. Polinização e dispersão de sementes em Myrtaceae do Brasil. Revista Brasileira de Botânica 29: 509-530.

Harper, J.L. 1977. Population biology of plants. London, Academic Press.

Iossi, E.; Sader, R.; Pivetta, K.F.L. \& Barbosa, J.C. 2003. Efeitos de substratos e temperaturas na germinação de sementes de tamareira-anã (Phoenix roebelenii O'Brien). Revista Brasileira de Sementes 25: 63-69.

Ista. 1985. International rules for seed testing. Seed Science and Technology 13: 356-513.

Maguire, J.D. 1962. Speed of germination-aid in selection and evaluation for seedling emergence and vigor. Crop Science 2: 176-177.

Masetto, T.E.; Davide, A.C.; Faria, J.M.R.; Silva, E.A.A. \& Rezende, R.K.S. 2009. Avaliação da qualidade de sementes de Eugenia pleurantha (MYRTACEAE) pelos testes de germinação e tetrazólio. Agrarian 2: 33-46.

Mayer, A.M. \& Poljakoff-Mayber, A. 1989. The Germination of Seeds. Oxford, Pergamon Press.

Mello, J.I.O. \& Barbedo, C.J. 2007. Temperatura, luz e substrato para a germinação de sementes de pau-brasil Caesalpinia echinata Lam., Leguminosae-Caesalpiniodeae. Revista Árvore 31: 645-655.

Norden, N.; Daws, M.I.; Antoine, C.; Gonzalez, M.A.; Garwood, N.C. \& Chave, J. 2009. The relationship between seed mass and mean time to germination for 1037 tree species across five tropical forests. Functional Ecology 23: 203-210.

Oliveira, L.M.; Carvalho, M.L.M.; Silva, T.T.A. \& Borges, D.I. 2005. Temperatura e regime de luz na germinação de sementes de Tabebuia impetiginosa (Martius ex A. P. de Candolle) Standley e T. serratifolia Vahl Nich. - Bignoniaceae. Revista Ciência e Agrotecnologia 29: 642-648.

Pedro Junior, M.J.; Brunini, O.; Alfonsi, R.R. \& Angelocci, L.R. 1977. Estimativa de graus-dia em função de altitude e latitude para o estado de São Paulo. Bragantia 36: 89-92.

Pimenta, R.S.; Luz, P.B.; Pivetta, K.F.L.; Castro, A. \& Pizetta, P.U.C. 2010. Efeito da maturação e temperatura na germinação de sementes de Phoenix canariensis hort. ex Chabaud - Arecaceae. Revista Árvore 34: 31-38.

Pires, L.A.; Cardoso, V.J.M.; Joly, C.A. \& Rodrigues, R.R. 2009. Germinação de Ternstroemia brasiliensis Cambess. (Pentaphylacaceae) de Floresta de Restinga. Acta Botanica Brasilica 23: 57-66.

Renaut, J.; Hoffmann, L. \& Hausman, J.F. 2005. Biochemical and physiological mechanisms related to cold acclimation and enhanced freezing tolerance in poplar plantlets. Physiologia Plantarum 125: 82-94.

Santana, D.G. \& Ranal, M.A. 2004. Análise da germinação: um enfoque estatístico. Brasília, Universidade de Brasília.

Santos, C.M.R.; Ferreira, A.G. \& Áquila, M.E.A. 2004. Características de frutos e germinação de sementes de seis espécies de Myrtaceae nativas do Rio Grande do Sul. Revista Ciência Florestal 14: 13-20.

Smith, H. Light quality and germination: ecological implications. 1975. In: Heydecher, W. Seed ecology. London, Buttrworth.

Stockman, A.L.; Brancalion, P.H.S.; Novembre, A.D.L.C. \& Chamma, H.M.C.P. 2007. Sementes de ipê-branco (Tabebuia roseo-alba (Ridl.) Sand. - Bignoniaceae): temperatura e substrato para o teste de germinação. Revista Brasileira de Sementes 29: 139-143.

Vázquez-Yanes, C.R. \& Orozco-Segovia, A. 1990. Ecological significance of light controlled seed germination in two contrasting tropical habitats. Oecologia 83: 171-175.

Villa Nova, N.A.; Pedro Júnior, M.J.; Pereira, A.R. \& Ometto, J.C. 1972. Estimativa de graus-dia acumulados acima de qualquer temperatura base em função das temperaturas máxima e mínima. São Paulo, Caderno Ciência da Terra 30: 89-92. 